\title{
A new Electrical Theorem
}

This content has been downloaded from IOPscience. Please scroll down to see the full text. 1894 Proc. Phys. Soc. London 1365

(http://iopscience.iop.org/1478-7814/13/1/307)

View the table of contents for this issue, or go to the journal homepage for more

Download details:

IP Address: 130.15.241.167

This content was downloaded on 03/10/2015 at 00:31

Please note that terms and conditions apply. 


\section{A new Electrical Theorem.}

\section{By Thomas H. Blakesley, M.A.*}

THE very short paper which I shall read to the Society contains the account of a Theorem which, though admitting of easy proof, appears, so far as my inquiries have gone, to have hitherto escaped notice.

In order to state the matter briefly, it will be well to adopt the following definition :-

If in any system of conductors, however reticulated, two or more modes of disposition of sources of electromotive force produce in every part of the network the same current, such systems of disposition are called equivalent systerns. Then the theorem is as follows:- In any system of conductors, possessing seats of electromotive force at any number of points, if any of these sources be supposed to move continuously along the various bars of the conducting system, and, where a point of junction is encountered, each to become a seat of the same electromotive force in each of the newly encountered bars (of course leaving the resistance of the source behind), then the disposition at any moment is equivalent to that at any other moment, and therefore to the original disposition. [Of course the direction of the electromotive force must be carefully maintained the same: if it is towards the knot before crossing it, it must be away from the knot after passing it.] The proof need only refer to the passing of a knot point, for no one will doubt that if the sources only move in an unbranching portion of the conductor the currents in different parts of the system will remain the same.

Let therefore the source $e$ approach the point $A$ at which its path splits into $n$ other ways. In each of the $n$ bars suppose a source $e$ inserted as directed, then these $n$ alone must be equivalent to the single source before reaching $A$; for if the $n$ sources are reversed, the current due to these sources in every portion of the system is reduced to zero. The reversed $n$ sources would therefore alone produce currents in the system

* Read February 23, 1894.

vOL. XIII. 
equal numerically, but opposite in direction, to those produced by the single source. Hence it follows that the $n$ sources (not reversed) will produce the same current as the single source.

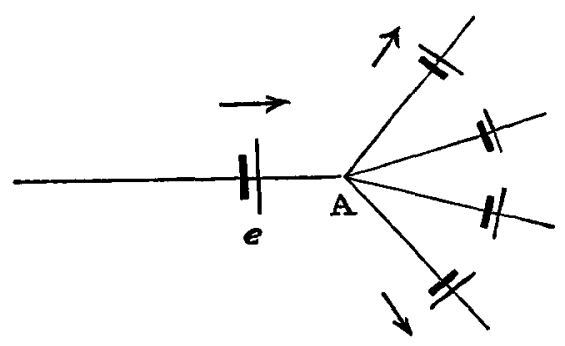

The principle of the superposition of currents enables us to apply this result to each source of the system, and therefore to prove the truth of the theorem in its complete generality.

In equivalent systems, since the current in every part remains the same, the total power expended remains the same; and equivalent systems might have been defined as those which produce the same expenditure of power in each part; and therefore the total power of the sources remains the same.

From the point of view of Kirchhoff's theorem $\Sigma e=\Sigma$.rc for any closed path in a network, the above general theorem may seem to some minds even plainer and more easily proved than on the method of demonstration which I have employed. For it is plain from the method of derivation, that if a seat of electromotive force exists in any closed Kirchhoff path it can never leave it ; and if in the movement of the sources one of them approaches the closed path under consideration, at the encounter it becomes in that path two equal sources acting in opposite directions.

If, therefore, $\Sigma e$ remains the same for any path and $r$ remains the same for every part, then obviously $c$ must remain the same for any portion of that path, and therefore for every part of the network.

The following propositions flow immediately from the main proposition :-

(1) If a closed continuous surface contains any region of any network, and some bar which cuts the surface contains, or by derivation as above can be made to contain, the seat of an 
electromotive force, then that source can- be done away with without disturbing the currents in any portion of the system provided that in the other bars which cut the surface sources of electromotive force be inserted of equal value but of opposite direction as regards inside and outside of the surface ; for it is clear that such sources would result from the migration of sources in one direction.

(2) If two systems of electromotive forces are equivalent, one may be derived from the other. For if system $\mathrm{A}$ is equivalent to system $B$, and we suppose $\frac{A}{2}$ to represent a distribution identical with $\mathrm{A}$ as regards positions, but of half the electromotive force in any case, then $\frac{A}{2}+\frac{B}{2}$ is equivalent to $A$ or $B$ alone. Now if any Kirchhoff path containing a source from $\frac{A}{2}$ does not also contain a source from $\frac{B}{2}$, then Kirchhoff's law would be outraged; for the sum of the electromotive forces in that path would be only half what they are from $A$ alone, whereas the currents and resistances remain the same. Hence for every Kirchhoff path there must be equal sources from each system. Either system may now have its elements moved up to those of the other system; any resulting side branchings will be the same (though differing in sign) whether derived from $\frac{A}{2}$ or from $\frac{B}{2}$, and must necessarily produce no effect by themselves, because if we consider $\frac{B}{2}$ to be reversed, the whole effect of $\frac{A}{2}$ and $\frac{B}{2}$, now in the same bars, will be zero. 